\title{
Empiric Communication Between an Expenditure and Other Descriptions of Stream of Water
}

\author{
Tatiana P. Melnyk* \\ Lviv Polytechnic University \\ 12 Bandera, Lviv, 79013, Ukraine
}

Received 20.12.2014, received in revised form 07.01.2015, accepted 30.01.2015

An area is inspected of empiric intercommunication of parameters of flow, finding of areas of the real and possible submergence out at passing of maximal charges, that can be a basis for the detailed spatial prognostication of flow of water and extrapolation of his motion in time for the r. Borzhava.

Keywords: empirical relationship, the average probability of error, extrapolation, mathematical models, mathematical modeling of runoff formation.

\section{Эмпирическая связь между расходом}

\section{и другими характеристиками потока воды}

\section{Т.П. Мельник}

Национальный университет «Львовская политехника» Украина, 79013, Львов, Степана Бандеры, 12

Исследована эмпирическая взаимосвязь параметров течения, выяснены зоны реального и возможного затопления при прохождении максимальных расходов, что может быть основой для детального пространственного прогнозирования стока воды и экстраполяции его хода во времени на примере р. Боржавы.

Ключевые слова: эмпирическая взаимосвязь, средневероятная погрешность, экстраполяиия, математические модели, математическое моделирование процессов формирования речного стока.

Закарпатской области необходимо уделять должное внимание для предупреждения воздействия паводковых вод и ликвидации последствий наводнений. Решение проблемы предотвращения стихийных бедствий во время наводнения в бассейне р. Боржава может быть, безусловно, обеспечено созданием множества прогностических систем, которые основаны на математическом моделировании процессов формирования речного стока. Здесь на поток воды влияет комплекс взаимосвязанных процессов, что в условиях горной местности осложняется

(C) Siberian Federal University. All rights reserved

* Corresponding author E-mail address: tatiana0508@ukr.net 
влиянием рельефа на пространственно-временное распределение метеорологических переменных. Благодаря уникальной системе автоматизированного наблюдения «Тиса» удалось за два дня предупредить местные органы власти области о возможном развитии чрезвычайных ситуаций и смоделировать возможные зоны затопления [1]. Как основание методической базы прогностической системы использованы математические модели формирования дождевого и снегодождевого стоков, а также производные от них - локальные модели [2-4]. Однако возникают потребности подробного пространственного прогнозирования стока воды и экстраполяции его течения во времени, что требует дополнительных методических решений.

На основе полученных материалов на участке проведения мониторинга $[5,6]$ произведены гидравлические расчеты прохождения паводков разной обеспеченности $(1,5,10 \%)$ и получены следующие характеристики: горизонты поверхностных вод, скорость течения в русле и на поймах (средние и максимальные) $[7,8]$. Расчетные уровни нанесены на продольном профиле. Это дало основу для размышлений, приведенных в статье.

Метод расчета паводков путем установления тех или иных эмпирических связей очень сложный. Суть его сводится к установлению связи между $\mathrm{Q}_{\max }$ и метеорологическими данными, такими как количество осадков, период снеготаяния, а в случае слива - период роста и спада волны паводка. Связь между первичными и вторичными факторами режима водного стока устанавливают в результате поиска. Они определяются уровнем воды $H=F(t)$, величиной паводка $Q=F(t)$, склоном $I=F(H)$, скоростью $V=F(H)$ и расходом $H=F(Q)$. При этом для каждого конкретного створа реки все гидрометрические кривые петлеобразны, потому что паводок распределяется сверху вниз по течению в виде паводковой волны. При этом в одном и том же сечении реки и при той же глубине потока на подъеме и спаде паводка вольная поверхность имеет разные наклоны. На подъеме паводка наклон вольной поверхности больше наклона долины, а на спаде наоборот. В связи с этим скорость течения расхода воды больше на подъеме паводка и меньше на спаде. Можно сделать вывод, что для каждого створа закономерна последовательность определенных максимальных значений гидрометрических характеристик паводка [9]:

$$
I_{\max } \rightarrow V_{\max } \rightarrow Q_{\max } \rightarrow H_{\max } .
$$

Между расходом и уровнями воды потока имеется гидравлическая связь. Если существуют расходы воды, каковы определены разными уровнями, легко установить зависимость $Q=Q(H)$ для определенного сечения потока воды. За существование уровней воды с помощью кривой расхода определяют расход $\mathrm{Q}$, не измеряя их [10]. Аналогично формируют $W=W(H)$ площадей живого сечения и средних скоростей $V=V(Q)$. Функции $Q=Q(H), W=W(H), V=V(H)$ связаны между собой равенством $Q=W^{*} V$.

Полученную зависимость $Q=Q(H)$ считают надежной, если средневероятная погрешность подсчитывается по формуле [11]

$$
\sigma= \pm 0,674 \sqrt{\sum(\Delta \sigma)^{2} / n},
$$

где $n$ - количество измерений расхода; $\Delta \sigma$ - отклонения, находящихся в пределах 2-4 \%.

Это дало основание для таких размышлений. В основание расчетов следует положить измеренные расходы воды в период свободного русла. Построить кривые $Q=Q(H)$ средних 
скоростей $V=V(H)$ и площадей водного сечения $W=W(H)$. В случае, когда данные $Q=Q(H)$, построенные по многолетним наблюдениям гидрологического поста, необходимо перенести на другой створ, задаются разные уровни воды для имеющегося створа $H_{0}$ и с помощью $Q=Q(H)$ определяют соответственные этим уровням расходы воды $Q$. Дальше по связи $H=H\left(H_{0}\right)$ устанавливают соответствующие уровни воды $H$ для расчетного створа. Определяем $Q=Q(H)$ для расчетного створа, предполагая, что при соответствующих уровнях воды расходы одинаковы.

Исходными данными для $Q=Q(t)$ являются ежедневные расчеты воды, которые определяем средними уровнями. Для стойкого ото льда русла ежедневные расходы воды рассчитываем непосредственно по зависимости $Q=Q(H)$. В зимний период расход воды сводится к расчету коэффициентов

$$
k_{3}=Q_{3} / Q_{6},
$$

где $Q_{3}$ - измеренные зимние расходы при уровне $H ; Q_{s}$ - летние расходы свободного русла при уровне $H$. Соответственно рассчитаем $k_{3}=k_{3}(t)$ и ежедневные расходы $Q_{3}=k_{3} * Q_{6}$. Аналогично определим $Q_{p}=k_{p} * Q_{b}$ из зависимости $k_{p}=k_{p}(t)$, где $Q_{p}$ - расход в заросшем русле при уровне $H ; Q_{6}$ - расход свободного русла при уровне $H$.

В случае деформации русла предположим, что $H$ - измеренный уровень, а $H_{c}$ - стандартный уровень, тогда погрешность до уровня рассчитывают формулой $\Delta H=H_{c}-H$, где имеется хронологическая зависимость $\Delta H=\Delta H(t)$ на конкретную дату.

Объем наполнения речной сети выражается формулой трапеций [11]:

$$
V(Q)=\frac{d t \cdot Q \cdot(1+R F)}{2(1-R F)},
$$

где $V(Q)$ - объем наполнения, $\mathrm{m}^{3} ; Q$ - расход воды в замыкающем створе, $\mathrm{m}^{3} / \mathrm{c} ; d t$ - часовой промежуток, с; $R F$ - коэффициент спада.

Погрешность оценки объема наполнения растет с увеличением временного промежутка суммирования $d t$. Определение оптимального промежутка времени зависит также от параметра $R F$, который относится к показателю продолжительности добегания воды:

$$
R F=f\left(\frac{L}{\sqrt{I}}\right)
$$

где $L$ - длина реки от истока, км; $I$ - средний уклон реки, \%о.

Значения $L / \sqrt{I}$ характеризуется формулой Шези - среднее время добегания воды от истока до замыкающего створа.

Экстраполировать кривую $Q=Q(H)$ с помощью формулы Шези уместно при наличии надежно измеренных наклонов поверхности воды для участков рек, в которых движение воды можно считать равномерным.

Для речных потоков эту формулу представляют в виде

$$
Q=w C \sqrt{h_{m t} I}
$$

где $h_{m t}=w / B-$ средняя глубина. 
Определим коэффициент Шези

$$
C=Q /\left(w / \sqrt{h_{m t} I}\right.
$$

и построим зависимость от уровня воды, т. е. $C=C(H)$.

Площадь водного сечения и средней глубины $h_{m t}$ во время высоких уровней есть в статистических данных, а наклон $I$ находим с помощью предварительно построенной графической зависимости $I=I(H)$.

Кривые $C=C(H)$ и $I=I(H)$ в своей верхней части имеют небольшую кривизну, их экстраполируем графически до необходимого высокого уровня воды. По данным $C$, $I$ и формулы (1) рассчитываем расход и строим кривую $Q=Q(H)$.

В борьбе с паводками осуществляют обвалование, высоту валов которого определяют наибольшим горизонтом воды. М.М. Гришин рекомендует $\Delta h$ определять, предполагая, что предельный наклон реки остается тем же, как и до обвалования, и что весь паводковый расчет проходит между валами. Для проверки пропускной способности русла можно использовать формулу Шези и предварительно задать $\Delta h$.

Для предварительного определения $\Delta h$ можно предположить, что расходы, которые проходят через обвалованные участки сечения: $q_{1}=w_{1} v_{1}$ и $q_{2}=w_{2} v_{2}$ на расстоянии $x_{1}+b_{3}+x_{2}$. Скорости на пойменных участках равны: $v_{1}=c_{1} \sqrt{h_{1} I}, v_{2}=c_{2} \sqrt{h_{2} I}, v_{3}=c_{3} \sqrt{h_{3} I}$, где $h_{1}, h_{2}, h_{3}-$ средние глубины, $c_{1}, c_{2}, c_{3}$ - коэффициент в формуле Шези.

Величину $c$ определяют по формуле Германека:

$$
\begin{aligned}
& c=30,7 \sqrt{h}, \text { если } h<1,5 м, \text { если } 1,5<h<6 м, \\
& c=50,2+0,5 h, \text { если } h>6 м .
\end{aligned}
$$

Предположим, что в слое $\Delta h$ скорость возрастет в сравнении со средними бытовыми на $25 \%$, тогда можно записать:

$$
\begin{aligned}
& w_{1} v_{1}+w_{2} v_{2}=1,25\left(x_{1} v_{1}+b_{3} v_{3}+x_{2} v_{2}\right) \Delta h_{1} . \\
& \Delta h=0,8 \frac{w_{1} v_{1}+w_{2} v_{2}}{x_{2} v_{2}+x_{1} v_{1}+b_{3} v_{3}} .
\end{aligned}
$$

Из формулы видно, что чем больше сжатие валами паводкового русла и чем меньше $x_{1}$ и $x_{2}$, тем $\Delta h$, а с ним и скорость течения больше и тем выше валы [11].

С.Н. Крицкий и М.Ф. Менкель (1956), используя уравнение Сен-Венана, также получили зависимость распространения паводковой волны течения потока для призматического русла. При выходе зависимости они отвергли инерционные члены; форму русла поперечного сечения предположили треугольной или прямоугольной, а гидрограф паводка - в виде треугольника во всех створах. Наклон поверхности воды на гребне волны рассчитывали равным наклону дна. Их зависимость для определения максимального расхода воды в нижнем створе $Q_{\text {max.н }}$, размещенном на расстоянии $x$ от верхнего створа, имеет вид

$$
Q_{\text {max. } . u}=Q_{\text {max.a }} \sqrt{\frac{1}{1+\frac{2 Q_{\max . b} n^{2}}{w^{2} i_{0}^{2}} x}},
$$


где $Q_{\max . d}, Q_{\max , u}-$ максимальный расход воды в верхнем и нижнем створах; $n$ - коэффициент жесткости по Маннингу; $W$ - общий объем паводковой волны [11].

Из формулы хорошо видно, что интенсивность распространения паводковой волны тем больше, чем острее гидрограф (чем больше отношение $\frac{Q_{\max . u}}{W}$ ), чем больше коэффициент $n$ и чем меньше наклон дна.

Сравним зависимости Д.И. Кочерина и Форхгеймера для паводка с гидрографом в виде треугольника при проточном затоплении, когда поток движется в пределах русла, не выходя на пойму. В формуле в числителе находится или отметка уровня, или величина расхода, что отвечает отрезку времени, на протяжении которого паводковая волна проходит по двойной волне определенного участка. Из сходств треугольников графика зависимости $Q$ от $t$ получим

$$
T=\frac{Q_{0}}{\delta Q_{0}} \frac{2 \Delta S}{C},
$$

где $T$ - период паводка.

Общий объем паводка определяем таким образом:

$$
W=\frac{1}{2} Q_{0} T=\frac{1}{2} Q_{0} \frac{Q_{0}}{\delta Q_{0}} \frac{2 \Delta S}{C} .
$$

Величина русловой емкости $R=B \Delta S H_{0} \approx B \Delta S \frac{Q_{0}}{c B}$.

Следовательно, величина распространения может быть получена непосредственно из гидрографа паводка $Q=f(t)$ одного поста. Для этого необходимо подсчитать величину $\frac{2 \Delta S}{C}$ и отложить ее на гидрографе параллельно оси времени.

Для определения расстояния между дамбами и их высоты использована зависимость

$$
L=l * \sqrt[3]{\left(\frac{b}{B}\right)^{2}},
$$

где $B$ - расстояние между дамбами; $b$ - ширина разлива; $L$ - глубина воды между дамбами; $l-$ средняя глубина затопления поймы.

До устройства дамб средняя скорость воды на пойме определяется как

$$
v=\frac{Q}{b l} .
$$

На обвалованном участке скорость будет:

$$
v_{1}=v \frac{b l}{B L} .
$$

Эта скорость не должна превышать допустимую, тогда размывания не будет.

Длину гряд $l_{\Gamma}, M$, при постоянном режиме движения воды определим зависимостью

$$
l_{\Gamma}=H \sqrt[3]{C^{2} / g},
$$

где $C$ - коэффициент Шези рассчитаем по вертикали при среднем значении наклона потока по ширине реки, $\mu^{0,5} / \mathrm{c} ; H$ - глубина потока на вертикали, $\boldsymbol{M} ; g=9,81 \mathrm{M} / \mathrm{c}^{2}-$ ускорение свободного падения.

$$
-121-
$$


Высоту гряд, $h_{\Gamma}, \mu$, следует определить зависимостями $h_{\Gamma}=0,25 H$ при $H<1 \mathrm{M} ; h_{\Gamma}=0,2+0,1 H$ при $H>1$. .

Скорость смещения гряд, $C_{\Gamma}, M / c$ определим по формуле

$$
C_{\Gamma}=0,019 \mathrm{~V} \cdot \mathrm{Fr}^{3},
$$

где $V$ - средняя скорость потока над местом определения гряды;

$$
\mathrm{Fr}=\mathrm{V} / \sqrt{\mathrm{gH}}-\text { число Фруда. }
$$

Период движения гряд постоянного профиля за сутки определяется формулой

$$
\tau_{\Gamma}=l_{\Gamma} / C_{\Gamma},
$$

где $l_{\Gamma}$ рассчитываем зависимостью $(1)$; $C_{\Gamma}$ - номограммами, $м / c y m$.

Ю.А. Ибад-Заде (1965), предполагая неизменность наклона потока в русле до и после возведения дамб, получил зависимость, которая связывает величину $\Delta h$ со скоростью и геометрическими характеристиками потока:

$$
\Delta h=\frac{\left(B_{1}-x\right) t_{1} v_{1}+\left(B_{2}-x_{2}\right) t_{2} v_{2}}{x_{1} v_{1}^{\prime}+B_{3} x_{3}^{\prime}+x_{2} v_{2}^{\prime}},
$$

где $\Delta h$ - повышение уровня в результате возведения дамб; $v_{1}, v_{2}, v_{3}$ - средняя скорость на пойме и в русле до возведения дамб; $v_{1}^{\prime}, v_{2}^{\prime}, v_{3}^{\prime}$ - средняя скорость на пойме и в русле после возведения дамб; $b_{1}, b_{2}, b_{3}, x_{1}, x_{2}$ - геометрическая характеристика потока пойм и русла.

Чем сильнее заужено русло дамбы, т.е. чем меньше $x_{1}$ и $x_{2}$, тем больше величина повышения уровня $\Delta h$, высота валов и скорость в пространстве между валами. Величина $\Delta h$ должна быть такой, чтобы не было разлива русла.

А.Ф. Печкуров (1964) предлагает для расчета использовать зависимости изменения глубины русла, полученные им из условий постоянных расходов потока и расходов наносов вдоль заданного участка реки и расширения или сжатия потока:

$$
H_{1}=H_{0}\left(\frac{B_{0}}{B_{1}}\right)^{0,75}
$$

где $H_{0}$ и $B_{0}$ - глубина и ширина потока до расширения; $H_{1}$ - глубина потока при расширении $A_{1}$.

Эта зависимость справедлива и для равномерного потока.

При установленном неравномерном, медленно переменном движении воды в открытом русле им предложена формула

$$
H_{1}=H_{0} \sqrt[5]{\left(\frac{B_{0}}{B_{1}}\right)^{2}} .
$$

Величина заиления расширенного участка $\Delta H=H_{0}-H_{1}$, соответственно, величина размыва для суженного участка $\Delta H=H_{1}-H_{0}[11]$.

С.Н. Корюкин принял те же начальные условия, что и С.Т. Алтунин. Он выразил расход воды $Q$ зависимостью Шези в таком виде [11]: 


$$
Q=w C \sqrt{R I} \approx w C \sqrt{h I}=w_{1} c_{1} \sqrt{h_{1} I}+w_{2} c_{2} \sqrt{h_{2} I}+w_{3} c_{3} \sqrt{h_{3} I}
$$

где $w$ - площадь поперечного сечения русла и поймы; $c$ - скоростной коэффициент; $h$ - средняя глубина в русле с поймой; $I$ - наклон потока; $w_{1}, w_{2}, w_{3}, C_{1}, C_{2}, C_{3}, h_{1}, h_{2}, h_{3}$ - соответственные величины для каждого участка поймы и русла.

Расход в пространстве между дамбами после их возведения $Q=w_{0} c_{0} \sqrt{R_{0} I} \approx w_{0} c_{0} \sqrt{h_{0} I}$, где $w_{0}$ - площадь поперечного сечения обвалованного русла при $h_{0}=\left(h_{3}+\Delta h\right)$ будет

$$
\begin{aligned}
& Q=w_{0} C_{0} \sqrt{h_{0} I}=B_{0} h_{0} C_{0} \sqrt{h_{0} I} \\
& \text { или } B_{0}=\frac{Q}{h^{\frac{3}{2}} \sqrt{I}} .
\end{aligned}
$$

Величину $\Delta h$ можно определить следующим образом [11]:

$$
\Delta h=h_{0}-h=h\left(\sqrt[3]{\left.\frac{B^{2}}{B_{0}^{2}}-1\right)} .\right.
$$

Задавая величину $B_{0}$ программными средствами Delphi, исследовали зависимость $\Delta h$. На основании результатов можно сделать вывод о необходимости отдаления дамб.

Если от русла с поймой отдалить дамбой часть поперечного сечения, то паводковая волна на этом участке будет проходить с увеличением расхода (или отметками), чем при полной емкости.

Расход, равный разнице между расходом повышенной волны паводка и бытовой, движется вдоль течения в виде «шапки», что накладывается на расходы всех постов, также разливается.

Величину разливания дополнительного расхода можно получить по формуле

$$
\frac{\Delta Q_{\max }^{\prime}-\Delta Q_{\max }}{Q_{\max }^{\prime}}=\frac{1}{z}\left(\delta^{*} z^{\prime}-\delta^{*} z \frac{Q_{\max }}{Q_{\max }^{\prime}}\right)
$$

Если допустить для упрощения, что $Q_{\max }^{\prime}=Q_{\max }$, то получим

$$
\frac{\Delta Q_{\max }^{\prime}-\Delta Q_{\max }}{Q_{\max }^{\prime}}=\frac{\delta^{*} z^{\prime}-\delta^{*} z}{z} .
$$

Приведенные зависимости установлены относительно одной точки гребня паводковой волны. Для определения характера трансформации волны паводка на любом участке должны быть применимы более точные методы расчета [11].

Расчет измерения волны методом псевдоустановленного движения или мгновенных режимов допускает предположения, которые были использованы в инженерных методах расчета, решать задачу относительно выравнивания паводковой волны. Здесь динамическое уравнение Сен-Венана сведено к зависимости Шези, какое может быть представлено в виде кривой объемов $W=f(H)$ или расхода $W=f_{1}(Q)$, где $W$ - объем (емкость) русла на участке; $H$ - средний уровень воды на участке в некоторый момент времени; $Q$ - средний расход на участке в этот же момент.

$$
-123-
$$


Замену будем считать правомерной, так как зависимость Шези равносильна кривой расходов, а кривую расходов можно заменить кривой объемов, так как каждому уровню или расходу на участке наблюдений отвечает определенный идентичный объем. Уравнение непрерывности при этом методе расчета будет иметь вид

$$
\bar{Q}_{1}-\overline{Q_{2}}=\frac{W}{\Delta t},
$$

где $\overline{Q_{1}}$ - средний расход входного створа за интервал времени $\Delta t ; \overline{Q_{2}}-$ средний расход в верхнем створе за интервал времени $\Delta t ; W$ - увеличение объема за это время.

Если обозначить величины, которые относятся к началу интервала времени $\Delta t$, звездой внизу, а величины, которые относятся к концу этого интервала, звездой сверху, то уравнение непрерывности будет иметь вид

$$
\frac{Q_{1^{*}}+Q_{1}^{*}}{2}-\frac{Q_{2^{*}}+Q_{2}^{*}}{2}=\frac{W^{*}-W_{*}}{\Delta t} .
$$

Этот способ расчета разрешает получить данные о трансформации всей паводковой волны (независимо от характера гидрографа). Русловой объем устанавливается на участках как функция уровня или соответственно среднему расходу в определенном промежутке времени:

$$
W_{*}=f\left(\frac{Q_{1^{*}}+Q_{2^{*}}}{2}\right)=f\left(Q_{*}\right)
$$

Анализ полученных данных позволяет определить зоны и уровень затопления поймы p. Боржавы паводками разной обеспеченности.

В данном исследовании установлена эмпирическая взаимосвязь параметров течения на примере бассейна р. Боржавы. Это позволит осуществлять расчеты гидравлических параметров минимальных и максимальных расходов, определять глубины и скорость, на основе чего выделить участки русла реки с минимальными глубинами, зоны затопления поймы, транзитную и аккумулирующую.

Осуществлять подсчеты целесообразно во время максимальных уровней воды, когда непосредственные измерения расхода невозможны или проблематичны, например, в период паводка. Кроме того, кривые $Q=Q(H)$ экстраполируют для проектирования гидротехнических сооружений, когда проектные уровни воды превышают высокие во время наблюдений.

\section{Список литературы}

[1] ua-REPORTER.com. Вiд стихії на Закарпатті найбільше постраждали Тячівський та Рухівський райони - сесія облради // Дані управління інформації та зв’язків із громадськістю Закарпатської облдержадміністрації від 30.07 .2008 р.

[2] Мельник Т.П. // Materialy II Miedzynarodowej naukowi-praktycznej konferencji «Perspektywiczne opracowania nauki i techniki - 2007». Tym 13. Rolnictwo. Chemia I chemiczne technologie. Ekologia. Geografia I geologia.: Przemysl. Nauka I studia S.77-82. 
[3] Мельник Т.П.. // Materialy IV mezinarodni vedecko - prakticka konference "Klicove aspekty vedecke cinnosti - 2008". -Dil 9. Matematika. Fyzika. Moderni informacni technologie: Praha. Publishing House "Education and Scince" s.r.o. S.37-41.

[4] Лук'янеиь O.I., Сусідко М.М. // Наук. праці УкрНДГМІ, 2004, Вип. 253

[5] Приплесь А.Й., Зубач В.М., Мельник Т.П. // Materialy IV miezynarodowej naukowipraktycznej cznei konferencji "Naukowym progres na rubiezy tesiacleci-2008" Tym 17. Chemia I chemiczne technologie. Ekologia. Geografia I geologia.: Przemysl. Nauka I studia. S. 72-76.

[6] Приплесь А.Й., Щучак М.Д. Мельник Т.П. // Materialy IV miezynarodowej naukowi praktycznei konferencji “Aktualne problemy nowoczesnych nauk-2008” Tym 20. Chemia I chemiczne technologie. Ekologia. Geografia I geologia.Weterynaria: Przemysl. Nauka I studia. S. 83-86.

[7] Якушев А.І., Зубач В.М., Мельник Т.П. Гідроморфологічний моніторинг стоку річок басейну р. Тиси і її приток. . - Рівне: Волинські обереги, 2009. 64 с.

[8] Мельник Т.П. Гідрогеолого-меліоративна оцінка р. Тиси в межах м. Рахів. Рівне: Волинські обереги, 2008. 92 с.

[9] Федотов Г.А. Изыскания и проектирование мостовых переходов. М.: Издательский центр «Академия», 2005. $304 \mathrm{c}$.

[10] Овчаров Е.Е., Захаровская Н.Н., Прошляков И.В. и др. Практикум по гидрологии, гидрометрии и регулированию П69 стока / ред. Е.Е. Овчаров. М.: Агропромиздат, 1988. 224 с.

[11] Чіпак В.П., Мельник Т.П. Система протипаводкових заходів у басейні р.Боржава. Рівне: Волинські обереги, 2008. 202 с. 